\title{
Profile of patients with idiopathic granulomatous mastitis presenting to a university teaching hospital in UAE: A case series
}

\section{Manda Venkatramana ${ }^{1,3}$, Pradeep Kumar Sharma ${ }^{1}$, Ghaith Jassim Jaber Al Eyd ${ }^{2,3}$}

${ }^{1}$ Department of Surgery, Gulf Medical College Hospital, ${ }^{2}$ Pathology and ${ }^{3}$ Gulf Medical

University, Ajman, UAE

Address for the Correspondence:

Prof. Manda Venkatramana,

Department of Surgery, Gulf Medical College Hospital, Ajman, UAE E-mail: researchdivision@gmu.

ac.ae

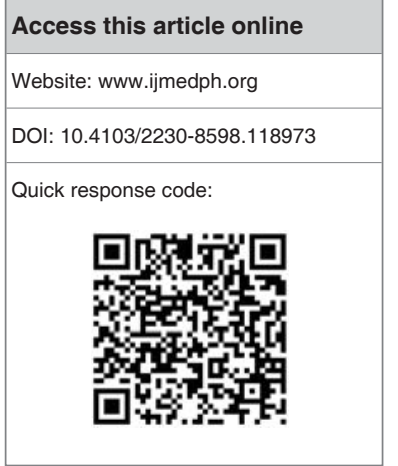

Objective: To highlight the profile of five patients diagnosed as having idiopathic granulomatous mastitis by histopathological examination. Materials and Methods: This presentation is a case series of patients, diagnosed as idiopathic granulomatous mastitis by histopathological examination of excised specimens. The total number of cases included in the report was five females over a 4-year period from 2005 to 2009. Results: The mean age of the patients was 29.6 years and the duration of symptoms varied from 3 weeks to 4 months. All the patients presented with a palpable mass in the breast, and two patients had associated pain and one had a bloody nipple discharge. The average size of the excised mass was $3.8 \times 3.1 \mathrm{~cm}$. Four out of the five patients diagnosed to have breast abscess or segmental mastitis had taken a course of antibiotics. One patient had a persistent wound sinus which subsided with ATT and the rest of the patients had no post-operative complications. Conclusion: Idiopathic granulomatous mastitis is a relatively rare disease which mimics the common breast disorders in the reproductive age group including malignancy.

Key words: Abscess, idiopathic, mastitis

\section{INTRODUCTION}

Idiopathic granulomatous mastitis (IGM) is a benign breast disease first described by Kesseler and Wolloch in 1972. ${ }^{[1]}$ Failure to recognize it can result in a diagnosis of cancer and the unnecessary performance of mastectomies. ${ }^{[2]}$ The disease is common in women in the child-bearing age group ${ }^{[3]}$ and in non-white females. ${ }^{[4-6]}$ The duration from the onset of symptoms to biopsy ranged from 3 weeks to 4 months, in the first reported cluster series of nine cases of IGM in Hispanic women during 2006-2008. Association of IGM with a history of recent pregnancy, breast feeding, and oral contraceptive pills has been reported. ${ }^{[7,8]}$ The present case series highlights the profile of five patients diagnosed as granulomatous mastitis by histopathological examination in a university teaching hospital in the UAE.

\section{MATERIALS AND METHODS}

This report is a case series of patients, diagnosed as IGM by histopathological examination of excised specimens over a 4-year period from 2005 to 2009.

\section{RESULTS}

The number of patients was five and the mean age was 29.6 years with the youngest being aged 24 years. The duration of symptoms varied from 3 weeks to 4 months. Three patients had a lesion in the right breast and the other two had lesions in the left. The location of the mass was at 9 o'clock position in two patients and at 1, 6, and 3 o'clock positions respectively, in the other three patients [Figure 1].

All the patients presented with a palpable mass in the breast. Two patients had associated pain and one had a bloody nipple discharge. All patients were anxious about the mass being a cancer. None of the patients in this series was either pregnant or lactating at the time of presentation. None of them was 
a smoker. The size of the excised masses ranged from $4.5 \times 3.5 \mathrm{~cm}$ to $2.5 \times 2.0 \mathrm{~cm}$, with an average size of $3.8 \times 3.1 \mathrm{~cm}$.

A clinical diagnosis of galactocele, fibrocystic, and segmental mastitis, respectively, was made in each of three patients, and breast abscess in the remaining two patients. Four out of the five patients diagnosed to have breast abscess or segmental mastitis had taken a course of antibiotics. One patient underwent ultrasound-guided wide bore aspiration with percutaneous biopsy of the abscess wall under local anesthesia. Two patients underwent I and D and biopsy of the abscess wall and the other two underwent excision biopsy of the mass under general anesthesia. Histopathological examination confirmed the diagnosis [Figures 2 and 3]. One patient had a persistent wound sinus which subsided with ATT and the others had no post-operative complications.

\section{DISCUSSION}

The five cases of granulomatous mastitis that reported to our hospital over a 4-year period were described in this report. The disease is common in women in the child-bearing age group, ${ }^{[3]}$ which is similar to our case series where the mean age is 29.6 years and the youngest being 24 years of age. The disease is common in nonwhite females which is similar to the findings in our series where all cases are nonwhite females. (1 from Pakistan and 4 Indians). ${ }^{[4-6]}$ The duration from the onset of symptoms to biopsy noted in our series ranges from as short as 3 weeks as long as 4 months which is similar to the first reported cluster series of nine cases of IGM in Hispanic women, during 2006-2008. Association of IGM with recent pregnancy, breast feeding and oral contraceptive pills has been reported,,$^{[7,8]}$ but in our series none of the patient's were pregnant, breast feeding or used oral contraceptive pills.

The treatment of choice for IGM has not yet been established. Complications of IGM seem to be related to the disease process and the surgical procedure and include skin ulceration, abscess formation, fistula, wound infection and recurrence. ${ }^{[9,10]}$ In our series two patients underwent I and $\mathrm{D}$ and biopsy of the abscess wall and the other two excision biopsy of the mass under general anesthesia as has been reported in literature. One patient had a persistent wound sinus which subsided with ATT and the rest of the patients had no post-operative complications. All patients had received a course of antibiotics and analgesics post-operatively.

The diagnosis of IGM was confirmed in all five cases based on histopathological findings during biopsies done to rule out malignancy.

\section{CONCLUSIONS}

Idiopathic granulomatous mastitis is a relatively rare disease which mimics the common breast disorders in the reproductive age group including malignancy. This causes anxiety and distress to the patient and needs histopathological examination to confirm the diagnosis.

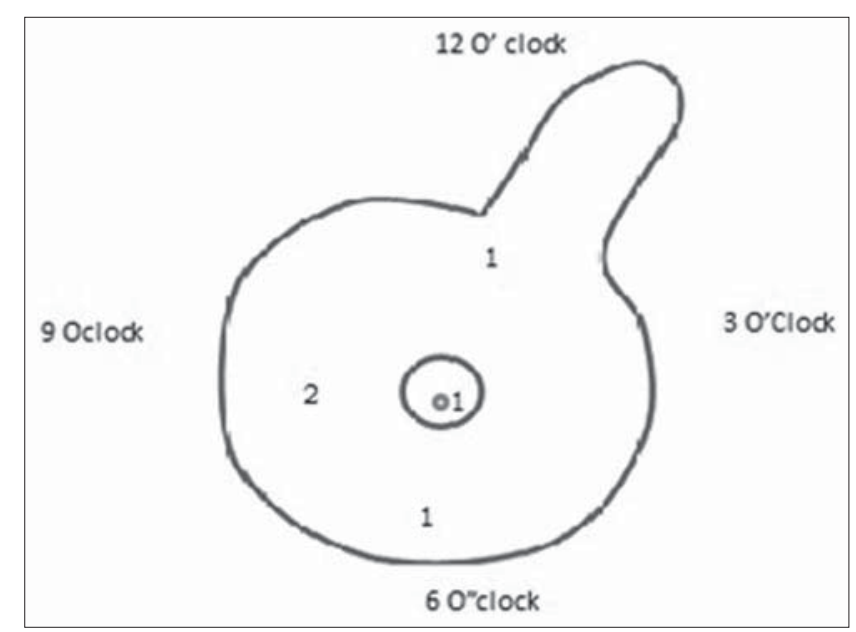

Figure 1: Location of lumps

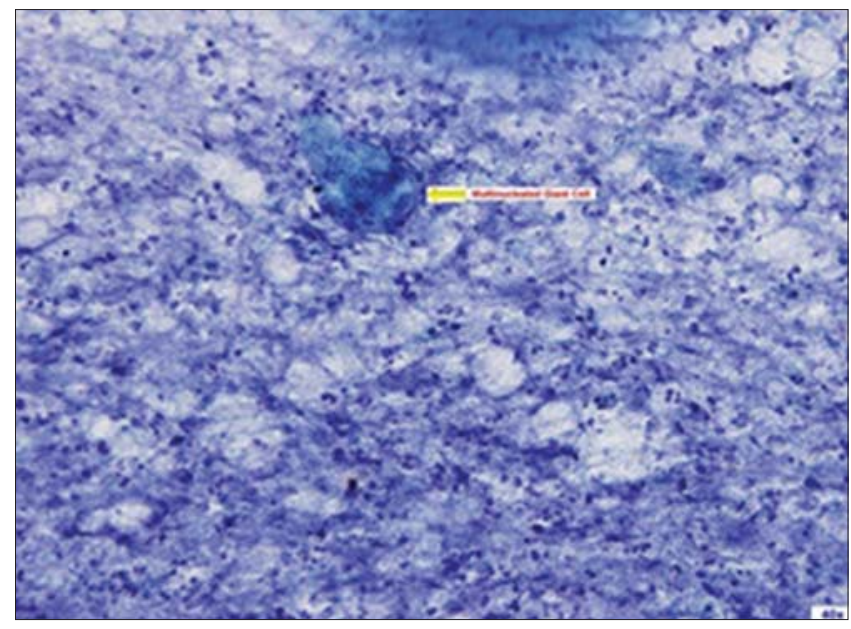

Figure 2: Multinucleated giant cell

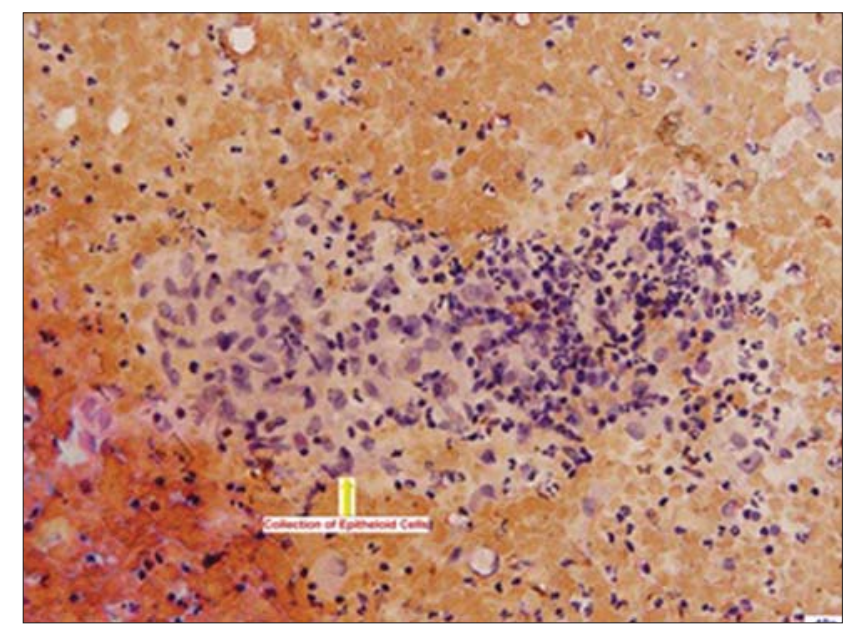

Figure 3: Collection of epitheloid cells

\section{REFERENCES}

1. Kesseler E, Wolloch Y. Granulomastitis: A lesion clinically simulating carcinoma. Am J Clin Pathol 1972;58:642-6.

2. Imoto S, Kitaya T, Kodama T, Hasebe T, Mukai K. Idiopathic granulomastitis: 
Case report and review of literature. Jpn J Clin Oncol 1997;27:274-7.

3. Tse GM, Poon CS, Ramhandran K, Ma TK, Pang LM, Law BK, et al. Granulomastitis: A clinical pathological review of 26 cases. Pathology 2004;36:254-7.

4. Baslaim MM, Khayat HA, Al-Amoudi SA. Idiopathic granulomastitis: A heterogeneous disease with variable clinical presentation. World J Surg 2007;31:1677-81

5. Al-Khaffaf B, Knox F, Bundred NJ. Idiopathic granulomastitis: A 25 years' experience. J Am Coll Surg 2008;206:269-72.

6. Aguirre-Gonzalez EH, Verduzco-Rodriguez L, Palet-Guzman JA. Granulomastitis mastitis. Report of 16 cases. Ginecol Obstet Mex 1999;67:509-11.

7. Going JJ, Anderson TJ, Wilkinson S, Chetty U. Granulomastitis lobular mastitis. J Clin Pathol 1987;40:535-40.
8. Aldaqal SM. Idiopathic granulomastitis. Clinical presentation, radiological features and treatment. Saudi Med J 2004;25:1884-7.

9. Fletcher A, Magrath IM, Riddell RH, Talbot IC. Granulomastitis: A report of seven cases. J Clin Pathol 1982;35:941-5.

10. Donn W, Rebbeck P, Wilson C, Gilks CB. Idiopathic granulomastitis: A report of three cases and review of the literature. Arch Pathol Lab Med 1994;118:822-5

How to cite this article: Venkatramana M, Sharma PK, Jaber Al Eyd GJ. Profile of patients with idiopathic granulomatous mastitis presenting to a university teaching hospital in UAE: A case series. Int J Med Public Health 2013;3:173-5.

Source of Support: Nil, Conflict of Interest: None declared. 